Supporting information:

\title{
Numerical calculation of apparent contact angles on hierarchical surface with array micro-structures by wire electrical discharge machining
}

Han Wang ${ }^{1}, 2$, Guanxin Chi ${ }^{1}$, Lei Li ${ }^{1}$, Sirui Gong ${ }^{1}$, Jialei Zhu ${ }^{1}$, Chuan Tian ${ }^{1}$, Yukui Wang ${ }^{1,2,{ }^{*}}$, Zhenlong Wang ${ }^{1,2,}{ }^{*}$

1 School of Mechatronics Engineering. Harbin Institute of Technology, Harbin 150001, Heilongjiang, China

2 Key Laboratory of Micro-systems and Micro-structures Manufacturing of Ministry of Education. Harbin Institute of Technology, Harbin Institute of Technology, Harbin 150001, Heilongjiang, China

${ }^{*}$ Corresponding authors

Yukui Wang

E-mail: wangyukui@hit.edu.cn

Zhenlong Wang

E-mail: wangzl@hit.edu.cn

Telephone: 0451-86413485 


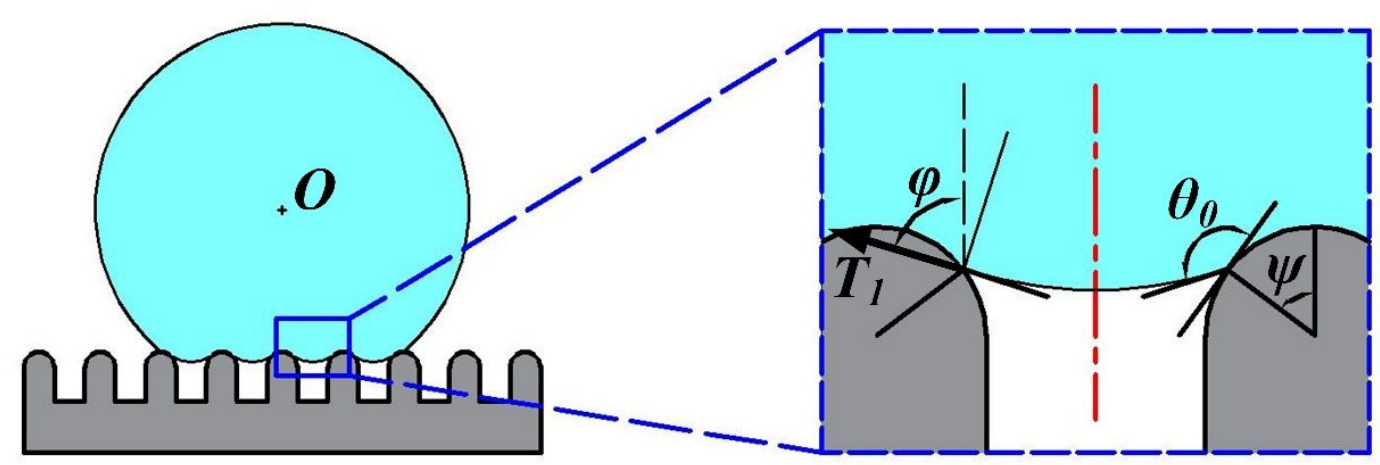

Figure.S1. The surface tension schematic diagram of water droplet in the interval with semicircle cross-section.

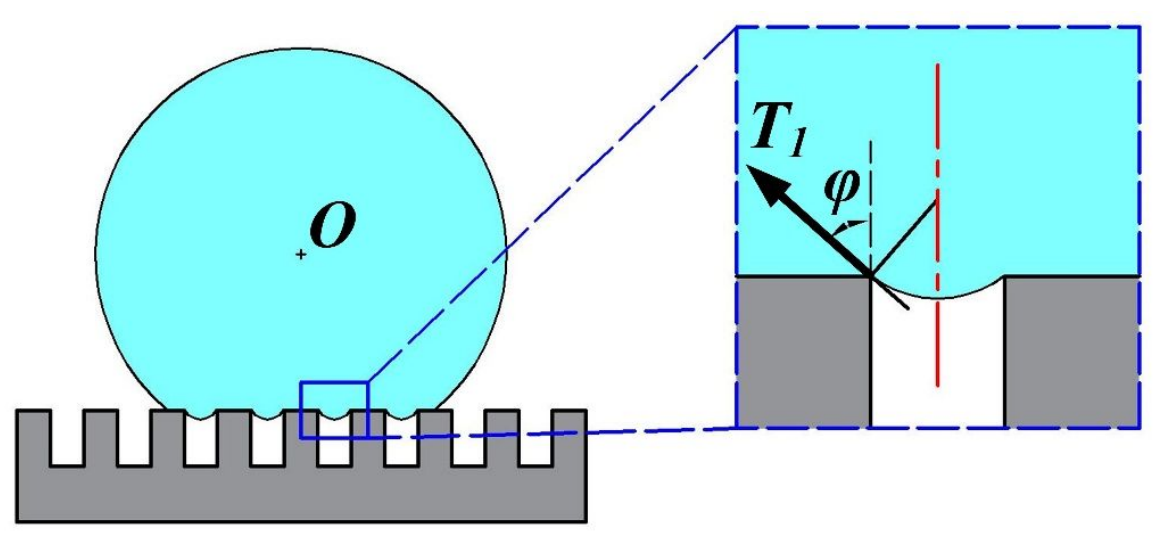

Figure.S2. The surface tension schematic diagram of water droplet in the interval with rectangle cross-section. 

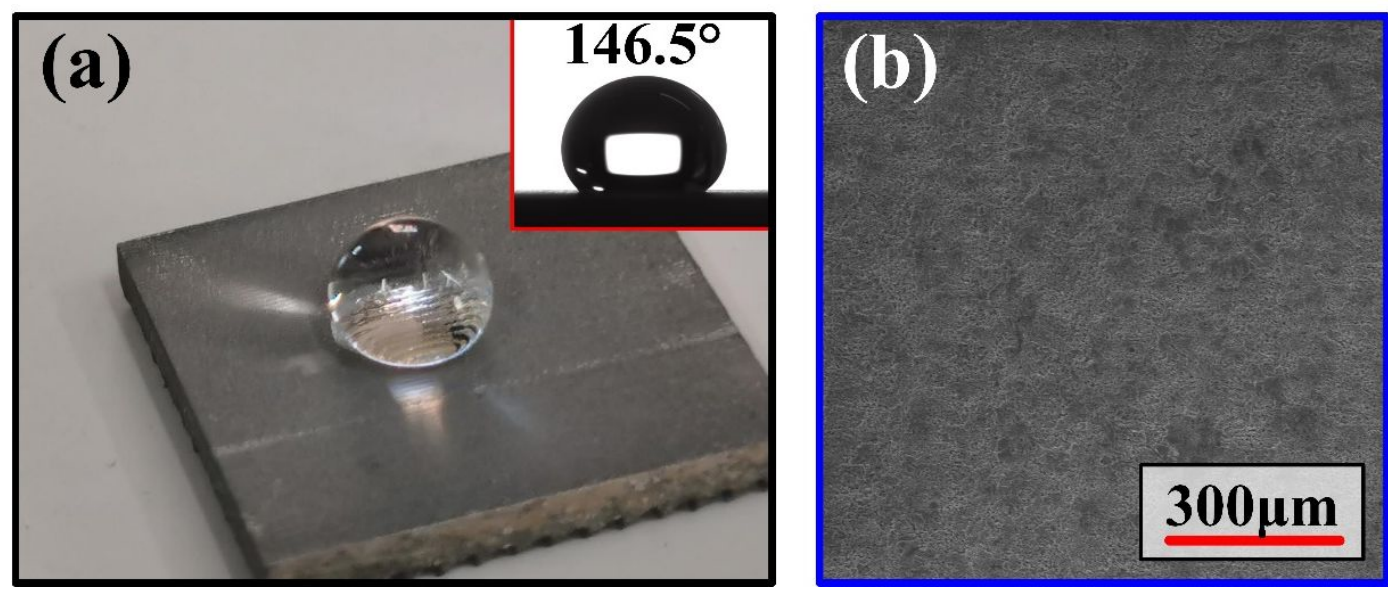

Figure.S3. (a) The digital photo of the water droplet on stearic acid treated WEDM surface and contact angle of water droplet. (b) The SEM image of rough WEDM morphology.
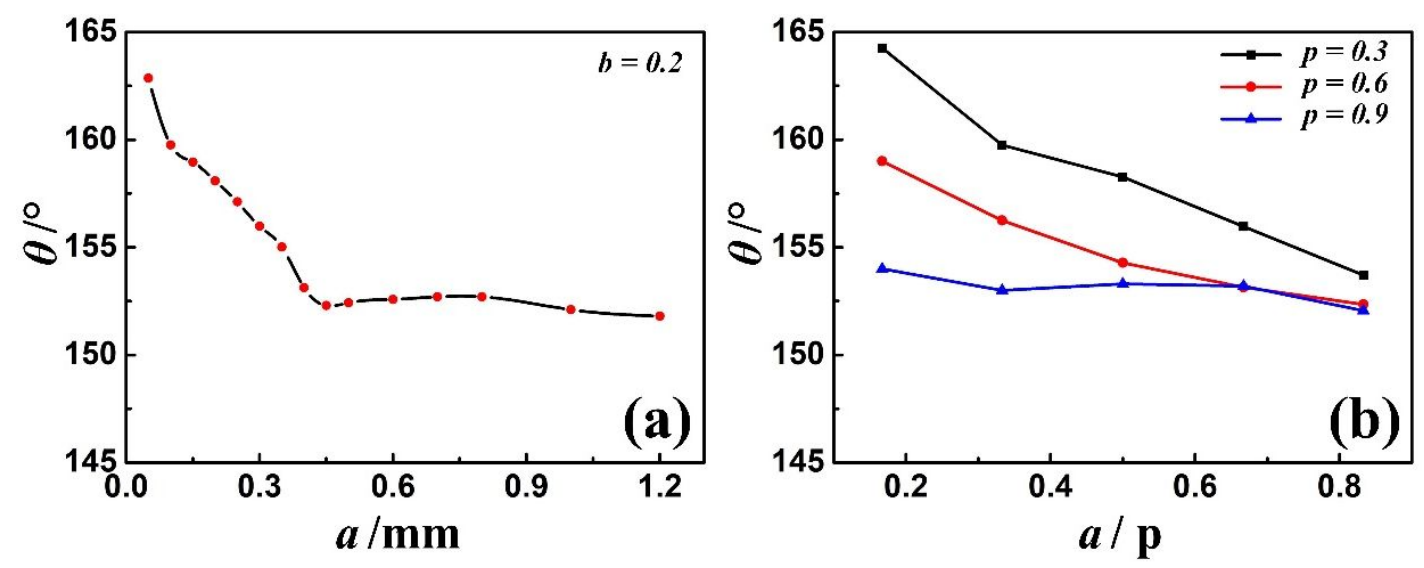

Figure.S4. The plots that effect of (a) width of micro-pillar $a$ and (b) ratio of width $a / p$ on apparent contact angle. 

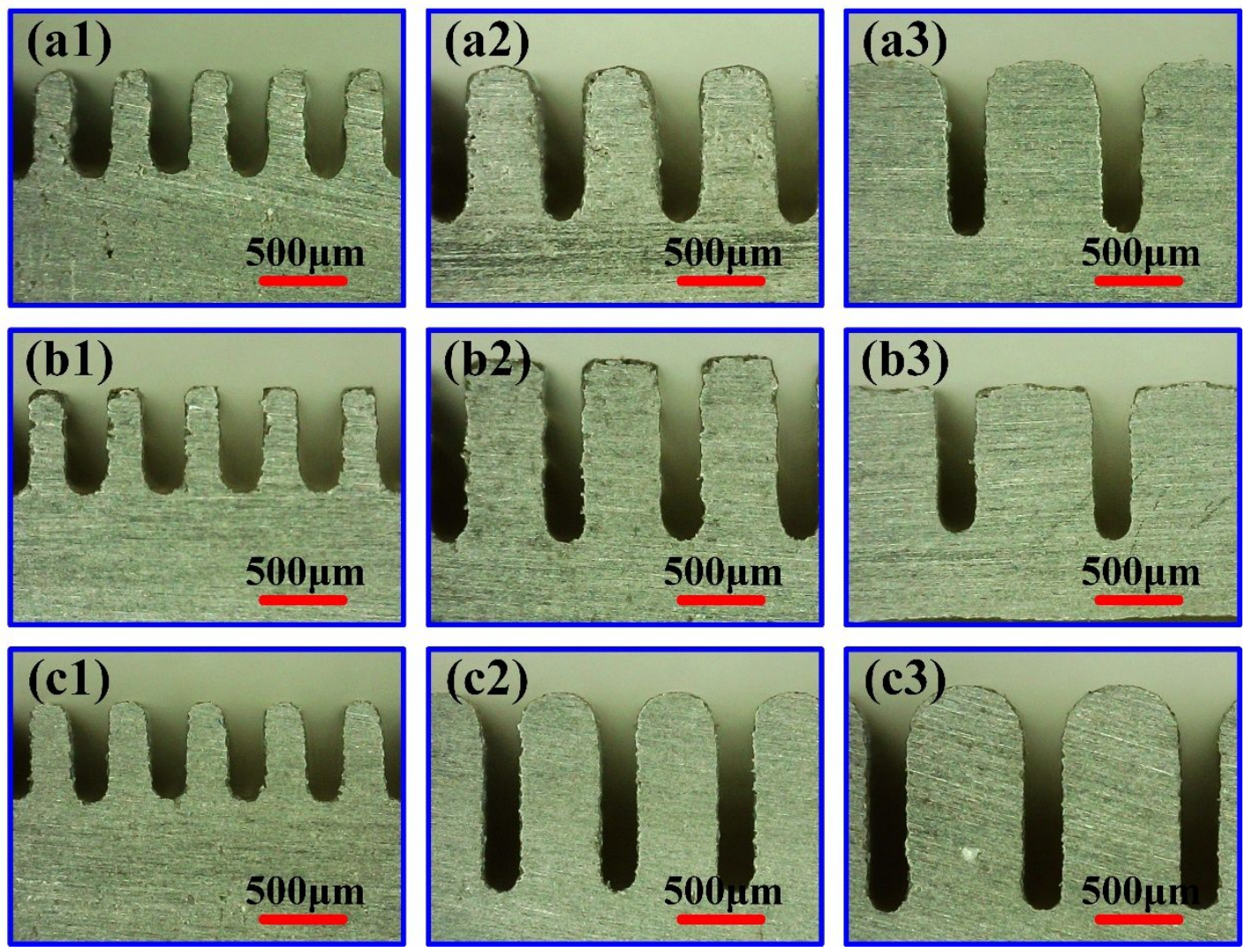

Figure.S5. Optical images of the side-view of array micro-pillars. (a) Rounded rectangle; (b) Rectangle; (c) Semicircle. Here $b=0.25 \mathrm{~mm},(\mathrm{a} 1)$ - (c1) $p=$ $0.5 \mathrm{~mm} ;(\mathrm{a} 2)-(\mathrm{c} 2) p=0.75 \mathrm{~mm} ;(\mathrm{a} 3)-(\mathrm{c} 3) p=1.0 \mathrm{~mm}$. 

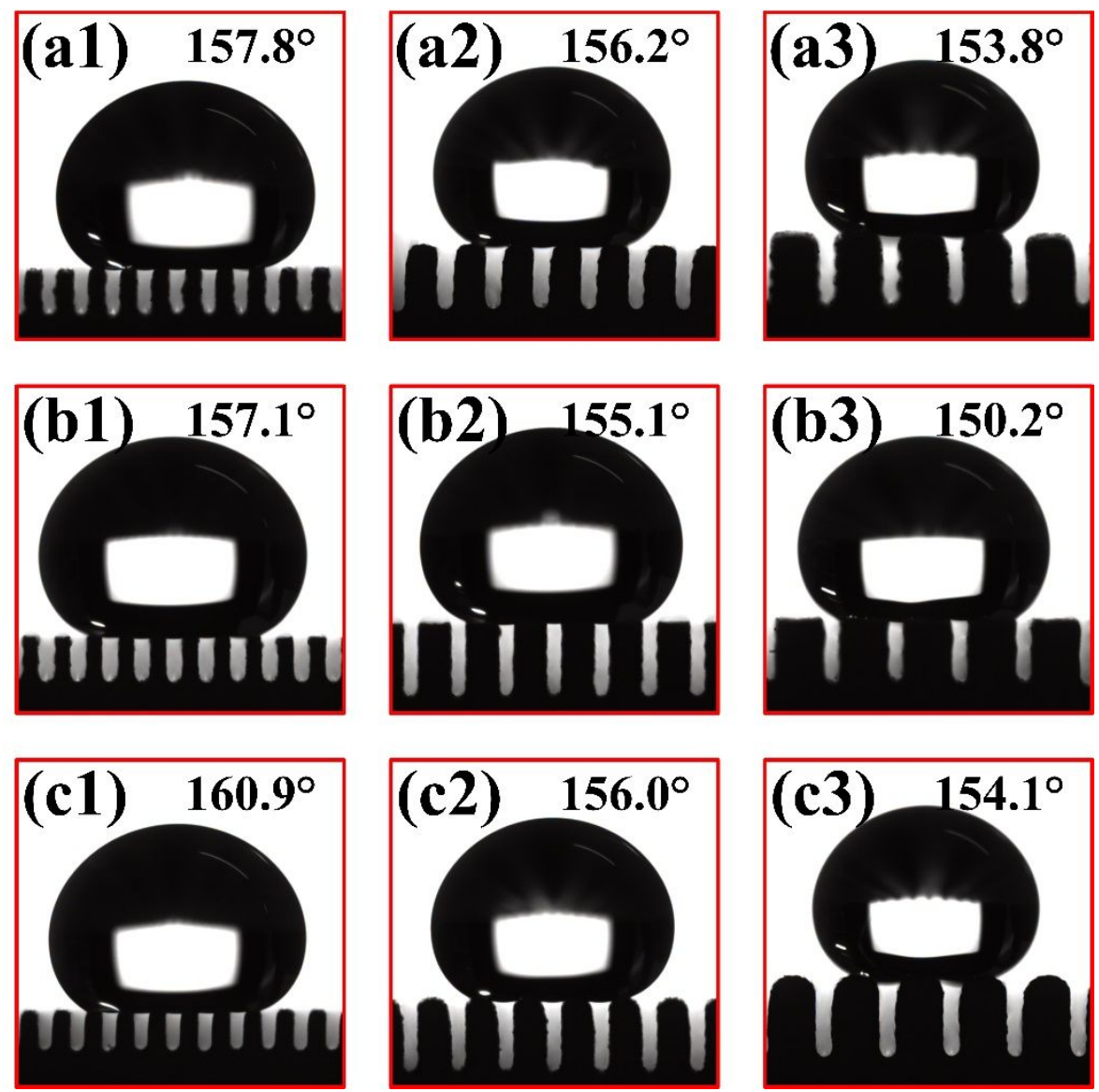

Figure.S6. Optical images of water droplets on array micro-pillars for contact angle measurement. (a) Rounded rectangle; (b) Rectangle; (c) Semicircle. Here $b=0.25 \mathrm{~mm}$, (a1) - (c1) $p=0.5 \mathrm{~mm}$; (a2) - (c2) $p=0.75 \mathrm{~mm}$; (a3) - (c3) $p=1.0 \mathrm{~mm}$. 

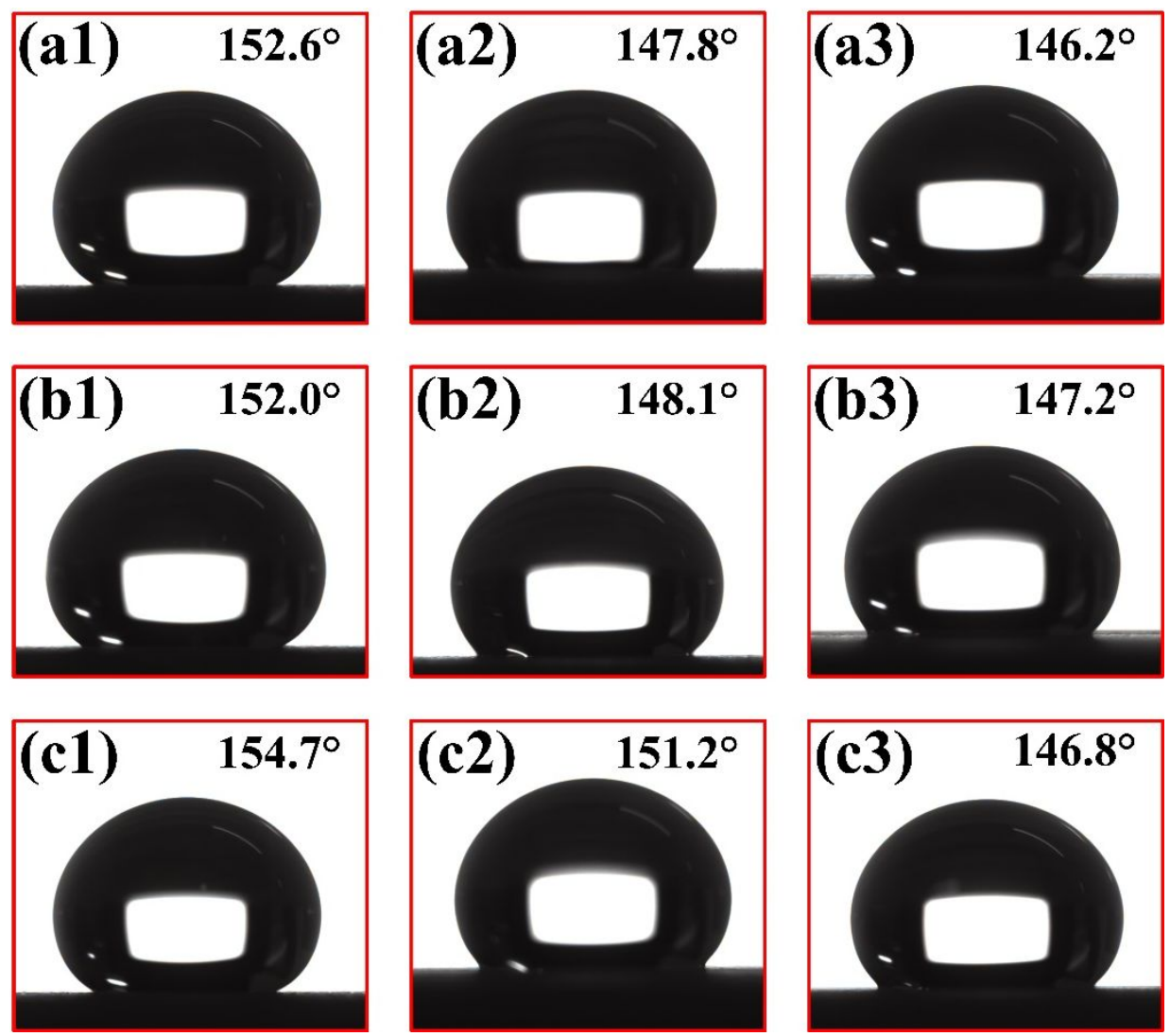

Figure.S7. Optical images of water droplets on array micro-pillars from side view. (a) Rounded rectangle; (b) Rectangle; (c) Semicircle. Here $b=0.25 \mathrm{~mm}$, (a1) - (c1) $p=0.5 \mathrm{~mm} ;(\mathrm{a} 2)$ - (c2) $p=0.75 \mathrm{~mm} ;(\mathrm{a} 3)$ - (c3) $p=1.0 \mathrm{~mm}$. 


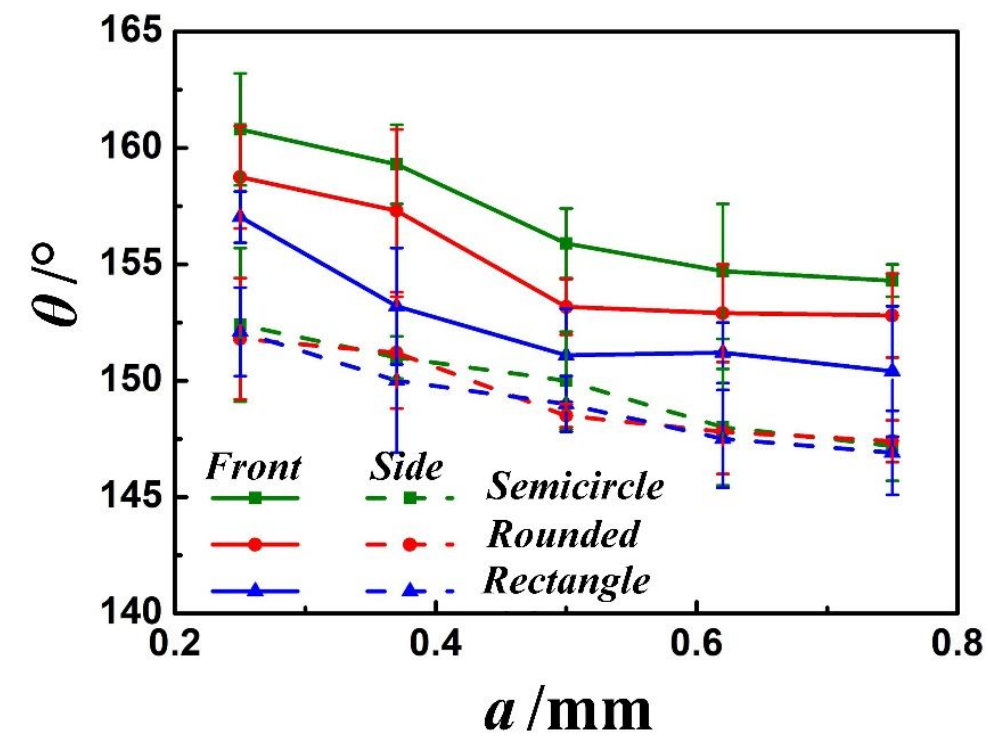

Figure.S8. The plots of measured contact angle varied with a on array microstructures by WEDM $(b=0.25 \mathrm{~mm})$ from front view and side view. 